\title{
Coexistence and Competition of Magnetism and Superconductivity on the Nanometer Scale in Underdoped $\mathrm{BaFe}_{1.89} \mathrm{Co}_{0.11} \mathrm{As}_{2}$
}

\author{
P. Marsik, ${ }^{1}$ K. W. Kim, ${ }^{1}$ A. Dubroka, ${ }^{1}$ M. Rössle,,${ }^{1}$ V. K. Malik, ${ }^{1}$ L. Schulz, ${ }^{1}$ C. N. Wang, ${ }^{1}$ Ch. Niedermayer, ${ }^{2}$ A. J. Drew, ${ }^{1,3}$ \\ M. Willis, ${ }^{3}$ T. Wolf, ${ }^{4}$ and C. Bernhard ${ }^{1, *}$ \\ ${ }^{1}$ University of Fribourg, Department of Physics and Fribourg Center for Nanomaterials, \\ Chemin du Musée 3, CH-1700 Fribourg, Switzerland \\ ${ }^{2}$ Laboratorium for Neutron Scattering, Paul Scherrer Institut \& ETH Zürich, CH-5232 Villigen, Switzerland \\ ${ }^{3}$ Queen Mary University of London, Mile End Road, London, E1 4NS, United Kingdom \\ ${ }^{4}$ Karlsruher Institut für Technologie, Institut für Festkörperphysik, D-76021 Karlsruhe, Germany
}

(Received 12 April 2010; published 27 July 2010)

\begin{abstract}
We report muon spin rotation $(\mu \mathrm{SR})$ and infrared spectroscopy experiments on underdoped $\mathrm{BaFe}_{1.89} \mathrm{Co}_{0.11} \mathrm{As}_{2}$ which show that bulk magnetism and superconductivity (SC) coexist and compete on the nanometer length scale. Our combined data reveal a bulk magnetic order, likely due to an incommensurate spin density wave (SDW), which develops below $T^{\mathrm{mag}} \approx 32 \mathrm{~K}$ and becomes reduced in magnitude (but not in volume) below $T_{c}=21.7 \mathrm{~K}$. A slowly fluctuating precursor of the SDW seems to develop already below the structural transition at $T^{s} \approx 50 \mathrm{~K}$. The bulk nature of SC is established by the $\mu$ SR data which show a bulk SC vortex lattice and the IR data which reveal that the majority of lowenergy states is gapped and participates in the condensate at $T \ll T_{c}$.
\end{abstract}

DOI: 10.1103/PhysRevLett.105.057001

The discovery of high temperature superconductivity (HTSC) in iron arsenide pnictides [1] has renewed interest in the relationship between magnetism and superconductivity (SC). Similar to the cuprate HTSC [2-4] and several heavy fermion superconductors [5], SC emerges here in close proximity to an antiferromagnetic (AF) or spin-density-wave (SDW) state [6-8]. Within the underdoped (UD) regime of the phase diagram it was even found that SC already sets in before static magnetism is suppressed [813]. These observations have reinforced speculation that the magnetic and SC orders are intimately related and that spin fluctuations may be at the heart of the SC pairing mechanism [14]. Nevertheless, it has been questioned whether the magnetic and SC orders truly coexist and interact on the nanometer scale. For example, it has been argued that they occur in adjacent but different regions of the phase diagram [15] and only coexist in spatially inhomogeneous samples with segregated magnetic and SC phases [16].

Here we present muon spin rotation ( $\mu$ SR) and infrared (IR) spectroscopy data which establish that in UD $\mathrm{BaFe}_{1.89} \mathrm{Co}_{0.11} \mathrm{As}_{2}$ the magnetic and $\mathrm{SC}$ orders are bulk phenomena which compete for the same electronic states.

$\mathrm{BaFe}_{1.89} \mathrm{Co}_{0.11} \mathrm{As}_{2}$ single crystals were grown from selfflux in glassy carbon crucibles. Their composition was determined by energy dispersive x-ray spectroscopy as described in Ref. [17]. The resistivity $\rho(T)$ and dc magnetization data yield a sharp SC transition at $T_{c}=21.7 \mathrm{~K}$ (see Fig. 1) that is consistent with a bulk SC state. The anomalous upturn in $\rho(T<50 \mathrm{~K})$ is characteristic of an UD sample with a structural transition at $T^{s} \approx 50 \mathrm{~K}$ and a magnetic one at $T^{\text {mag }} \leq T^{s}[8,18,19]$.
PACS numbers: 74.70.Xa, 74.25.Ha, 76.75.+i, 78.30.-j

The $\mu$ SR experiments on a mosaic of crystals were performed at the GPS instrument at the $\pi M 3$ beam line of the Paul Scherrer Institut (PSI) in Villigen, Switzerland. $\mu \mathrm{SR}$ measures the time evolution of the spin polarization $P(t)$ of the implanted muon ensemble using the asymmetry $A(t)$ of the decay positrons [20]. The technique is well suited to studies of magnetic and SC materials, as it allows a microscopic determination of the internal field distribu-
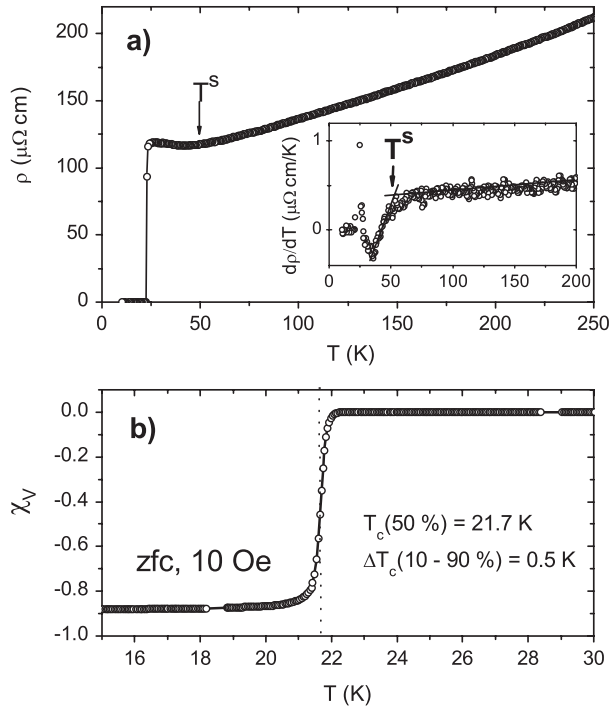

FIG. 1. (a) $T$ dependence of the in-plane resistivity $\rho$ and (b) the zero-field-cooled (zfc) magnetic volume susceptibility $\chi_{V}$ of $\mathrm{BaFe}_{1.89} \mathrm{Co}_{0.11} \mathrm{As}_{2}$ with $H=10$ Oe parallel to the $c$ axis. Inset: Derivative of $\rho(T)$ showing the change in slope around $T^{s} \approx 50 \mathrm{~K}$. 
tion and gives direct access to the volume fractions of these phases. The $100 \%$ polarized positive muons are implanted into the bulk of the sample stopping at interstitial lattice sites [21] within a 100-200 $\mu \mathrm{m}$ thick layer. Each muon spin precesses in the local magnetic field $B_{\mu}$ with a precession frequency of $\nu_{\mu}=\gamma_{\mu} \times B_{\mu} / 2 \pi$, where $\gamma_{\mu}=$ $2 \pi \times 135.5 \mathrm{MHz} / \mathrm{T}$ is the muons gyromagnetic ratio.

IR spectroscopy measurements were performed on $a b$-plane surfaces of freshly cleaved crystals from the same growth batch. The reflection and ellipsometry techniques and the data analysis are described in Ref. [22]. Since the probe depth of the IR light exceeds $100 \mathrm{~nm}$, the IR spectra yield the bulk electronic properties. In particular, they enable one to access the fraction of electronic states that participate in the SC condensate [22,23].

The results of the $\mu$ SR experiments are summarized in Fig. 2. The zero-field (ZF) $\mu$ SR spectra in Fig. 2(a) establish that static magnetism develops below $\sim 40 \mathrm{~K}$. They have been fitted (solid lines) with the function

$$
P(t)=P(0)\left[A_{f} \exp \left(-\lambda_{f}^{\mathrm{ZF}} t\right)^{\beta}+A_{s} \exp \left(-\lambda_{s}^{\mathrm{ZF}} t\right)\right],
$$

where the two terms account for the fast $(f)$ and slowly $(s)$ depolarizing parts of the signal, respectively. The rapid depolarization of $P(t)$ at low $T$ without a trace of an oscillation suggests that the magnetic state is either incommensurate or strongly disordered. The IR data shown below support an incommensurate spin density wave (SDW). The low- $T$ value of $\lambda_{f}^{\mathrm{ZF}} \approx 8 \mu \mathrm{s}^{-1}$ yields an estimate of the average local field of $\left\langle B_{\mu}^{\mathrm{ZF}}\right\rangle \approx \Lambda^{\mathrm{ZF}} / \gamma_{\mu} \approx$ $10 \mathrm{mT}$ which is around 5\% of the value in undoped
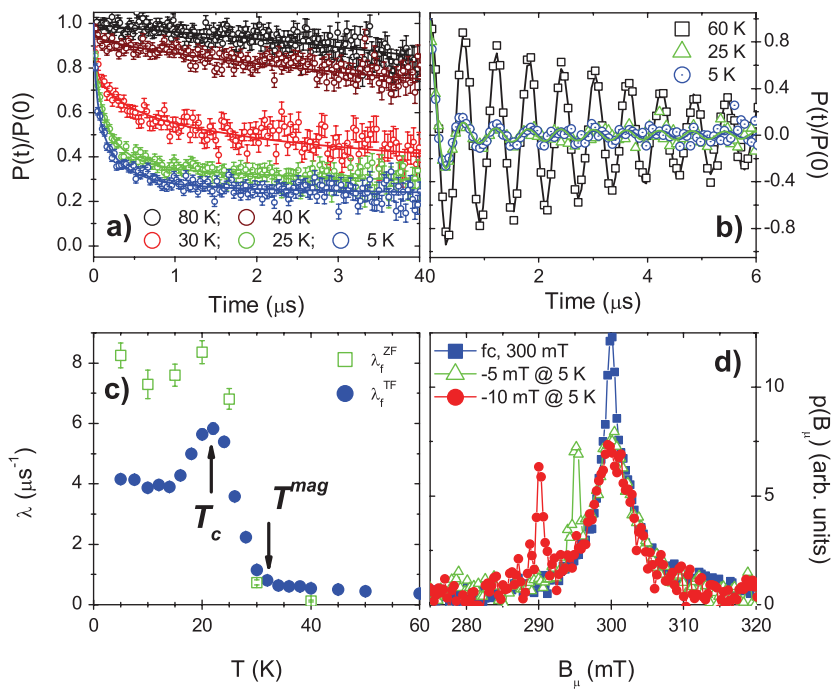

FIG. 2 (color). (a) ZF- $\mu$ SR time spectra (symbols) showing a rapid depolarization below about $40 \mathrm{~K}$. Solid lines show fits with Eq. (1). (b) TF- $\mu$ SR spectra (symbols) at $H_{\mathrm{ext}}=300 \mathrm{mT}$ plotted in a rotating reference frame $\left(\omega_{\mathrm{RRF}}=39.5 \mathrm{MHz}\right)$. Solid lines show fits with Eq. (2). (c) $T$ dependence of the depolarization rates $\lambda_{f}^{\mathrm{TF}}$ and $\lambda_{f}^{\mathrm{ZF}}$. (d) TF- $\mu \mathrm{SR}$ line shapes showing the distribution of local magnetic fields $p\left(B_{\mu}\right)$ during a so-called pinning experiment as described in the text.
$\mathrm{BaFe}_{2} \mathrm{As}_{2}$ [7,24]. The amplitude of $A_{f} \approx 0.7$ confirms that the magnetic volume fraction is at least $70 \%$. However, it is likely to be even higher since $\vec{B}_{\mu}^{\mathrm{ZF}}$ may not be orthogonal to $\vec{P}(t=0)$.

This magnetic volume fraction has been more accurately determined from the transverse-field (TF) $\mu$ SR data as shown in Fig. 2(b) where the direction of the external field of $H_{\text {ext }}=300 \mathrm{mT}$ is well defined. The TF- $\mu \mathrm{SR}$ spectra were fitted with the function:

$$
\begin{aligned}
P(t)= & P(0)\left[A_{f} \cos \left(\gamma B_{\mu, f} t\right) \exp \left(-\lambda_{f}^{\mathrm{TF}} t\right)\right. \\
& \left.+A_{s} \cos \left(\gamma B_{\mu, s} t\right) \exp \left(-\lambda_{s} t\right)\right] .
\end{aligned}
$$

At $100 \mathrm{~K}$ there is only a weak depolarization of the oscillatory signal which typically arises from nuclear moments. At 25 and $5 \mathrm{~K}$ the major part of the signal depolarizes very rapidly due to the presence of static electronic moments. At both temperatures we obtain the same amplitudes of $A_{f} \approx 0.92$ and $A_{s} \approx 0.08$ that are characteristic of bulk magnetism. The latter fraction is well accounted for by the background of muons that stop in the sample holder or the cryostat shields and windows. Our TF- $\mu$ SR data therefore provide compelling evidence for a magnetic phase that occupies more than 90\% (likely even 100\%) of the volume. This bulk magnetic state may be strongly disordered and exhibit a large spatial variation in magnitude. Nevertheless, since the magnetic stray fields away from regions with AF or SDW order are known to rapidly fall off on the scale of a few nanometers, any nonmagnetic regions must be very small and intermixed with the magnetic ones.

The $T$ dependence of the magnetic order is detailed in Fig. 2(c) in terms of the relaxation rates $\lambda_{f}^{\mathrm{ZF}}$ and $\lambda_{f}^{\mathrm{TF}}$. The magnetic transition at $T^{\mathrm{mag}} \approx 32 \mathrm{~K}$ is identified from the steep increase of $\lambda_{f}^{\mathrm{TF}}$. There is also a weak, yet noticeable increase of $\lambda_{f}^{\mathrm{TF}}$ above $T^{\mathrm{mag}}$ which is likely due to a fluctuating magnetic state as discussed below in the context of the IR data. The most remarkable feature is the anomaly at $T_{c}=21.7 \mathrm{~K}$ below which $\lambda_{f}^{\mathrm{TF}}$ decreases again. This observation agrees with previous neutron diffraction studies which revealed a similar SC-induced reduction of the intensity of a magnetic Bragg peak [10,11]. The neutron data exclude a reorientation of the magnetic moments or a change in their periodicity. However, they cannot distinguish between a SC-induced reduction of the order parameter amplitude and a decrease of the magnetic volume fraction [11]. Our $\mu$ SR data provide this additional information and thus help to identify the scenario of a SCinduced reduction of the magnetic order parameter.

Our $\mu$ SR data also confirm the bulk nature of SC. Figure 2(d) displays a so-called pinning experiment which demonstrates that a bulk SC vortex lattice develops below $T_{c}[4,25]$. It shows so-called $\mu$ SR-line shapes, as obtained from a fast-Fourier transformation of the TF- $\mu$ SR time spectra, which detail the distribution of the magnetic field probed by the muons, $p\left(B_{\mu}\right)$. The first measurement 
(squares) has been performed directly after field cooling (fc) the sample to $5 \mathrm{~K}$ at $H_{\text {ext }}=300 \mathrm{mT}$. Before the second measurement (triangles) $H_{\text {ext }}$ was reduced by 5 to $295 \mathrm{mT}$. Finally, $H_{\mathrm{ext}}$ was reduced by another 5 to $290 \mathrm{mT}$ before the third measurement (circles). The line shapes consist of a weak, narrow peak due to the background muons and a dominant, broad peak which arises from the muons that stop inside the magnetic sample. The weight of these peaks agrees with $A_{f} \approx 0.92$ and $A_{s} \approx 0.08$ as obtained from the time spectra. The striking result is that the broad part of the line shape remains fully pinned, while only the narrow peak is following the changes of $H_{\mathrm{ext}}$. This behavior highlights a complete pinning of the magnetic flux density in the sample which is the hallmark of a bulk type-II superconductor. While the pinning centers are regions where SC is reduced or even vanishing, for the SC vortices to be rigid and the pinning to be effective, the majority of the volume must be SC. The circumstance that the entire sample is at the same time magnetic, is manifested by the broadening of the $\mu$ SR line shape which is much larger than expected for a SC vortex lattice [26-28].

Our IR data in Fig. 3 confirm the bulk nature of SC. Similarly as previously reported for OP samples $[22,29,30]$, they reveal characteristic SC-induced changes due to the formation of a bulk SC state with several nearly isotropic BCS-like energy gaps [22]. Notably, the reflectivity, $R\left(T \ll T_{c}\right)$, approaches unity around $50 \mathrm{~cm}^{-1}$ and the real part of the optical conductivity, $\sigma_{1}\left(T \ll T_{c}\right)$, has a pronounced lower gap edge around $50 \mathrm{~cm}^{-1}$ below which it essentially vanishes. This highlights that the vast majority of the low-energy electronic states is captured by the SC energy gap(s) and participates in the SC condensate. The so-called Ferrell-Glover-Tinkham sum rule requires that the spectral weight, $\operatorname{SW}(\Omega)=\frac{120}{\pi} \int_{0}^{\Omega} \sigma_{1}(\omega) d \omega$, with

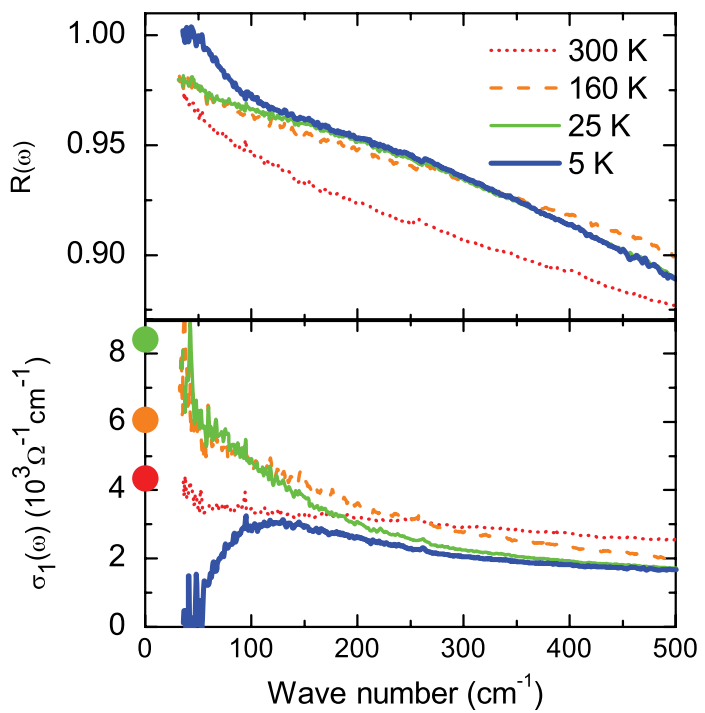

FIG. 3 (color online). $T$-dependent spectra of (a) the FIR reflectivity $R(\omega)$ and (b) the in-plane optical conductivity $\sigma_{1}(\omega)$ of $\mathrm{BaFe}_{1.89} \mathrm{Co}_{0.11} \mathrm{As}_{2}$ with $T_{c}=21.7 \mathrm{~K}$. Solid circles show $\sigma^{\mathrm{dc}}$ as obtained from Fig. 1(a). $\sigma_{1}(\omega)$ in units of $\Omega^{-1} \mathrm{~cm}^{-1}$, remains unaffected by the SC transition for $\Omega \geq 6-10 \Delta^{\mathrm{SC}}$ [31]. Accordingly, the socalled missing SW due to the gaplike suppression of the regular part of $\sigma_{1}$ is transferred to a $\delta$ function at $\omega=0$. Its weight, $\mathrm{SW}^{\delta}=4 \pi e^{2} \frac{n_{s}}{m^{*}}=\frac{c^{2}}{\lambda_{a b}^{2}}$, with the condensate density $n_{s}$, effective mass $m^{*}$, and magnetic penetration depth $\lambda_{a b}$, can be deduced either from the missing SW in $\sigma_{1}$, or likewise from the inductive response in the imaginary part with $\sigma_{2}^{\delta}(\omega)=\frac{c^{2}}{\omega \lambda_{a b}^{2}}[22,23]$. For our UD sample we obtain $\lambda_{a b} \approx 305(10) \mathrm{nm}$, which, assuming the same value of $m^{*}$, corresponds to a value of $n_{s}$ that is reduced by about $20 \%$ as compared to OP $\mathrm{BaFe}_{1.87} \mathrm{Co}_{0.13} \mathrm{As}_{2}$ with $\lambda_{a b} \approx 270 \mathrm{~nm}[22]$.

Figure 4 shows that our optical spectra also contain the signatures of the SDW state and its competition with SC. Similar to other SDW systems [32], including undoped $\mathrm{BaFe}_{2} \mathrm{As}_{2}$ [33], a part of the low-energy SW is transferred to a pair breaking peak (PBP) near the gap edge at $2 \Delta^{\mathrm{SDW}}$. This PBP shows up in Fig. 4(a) as a broad maximum in $\Delta \sigma_{1}=\sigma_{1}(25 \mathrm{~K})-\sigma_{1}(50 \mathrm{~K})$ for the UD sample. For the OP sample it is absent and $\Delta \sigma_{1}$ only reveals a narrowing of the Drude response. Assuming that in the UD sample the Drude response is subject to a similar narrowing, we subtracted this contribution to single out the changes due to the SDW. This reveals a broad maximum corresponding to $2 \Delta^{\mathrm{SDW}} \approx 200-300 \mathrm{~cm}^{-1}$ which, similar to $T^{\mathrm{mag}} \approx$ $32 \mathrm{~K}$ and $T^{s} \approx 50 \mathrm{~K}$, is reduced by $\sim 3-4$ times as compared to undoped $\mathrm{BaFe}_{2} \mathrm{As}_{2}$ with $2 \Delta^{\mathrm{SDW}} \approx 900 \mathrm{~cm}^{-1}$ and $T^{\mathrm{mag}}=T^{s}=138 \mathrm{~K}$ [33]. In agreement with the magnetic order parameter as deduced from $\mu \mathrm{SR}$, we obtain a SW of the broad maximum due to the PBP of $\sim 25000 \Omega^{-1} \mathrm{~cm}^{-2}$ which is around $5 \%$ of the value in $\mathrm{BaFe}_{2} \mathrm{As}_{2}$ [33].

The $T$ dependence of the PBP is detailed in Fig. 4(c), which compares the evolution of the SW between 600 and $1400 \mathrm{~cm}^{-1}, \mathrm{SW}^{600-1400}$, in the UD and OP samples. In the latter, $\mathrm{SW}^{600-1400}$ decreases monotonically towards low $T$ with no anomaly at $T_{c}$. For the UD sample, clear deviations occur at low $T$ which signify that the PBP gradually develops below $T^{s} \approx 50 \mathrm{~K}$. There is some extra increase below $T^{\text {mag }} \approx 32 \mathrm{~K}$, but this is rather small as compared to the one of $\lambda^{\mathrm{TF}}$ in Fig. 2(c). This discrepancy may be resolved in terms of a precursor SDW which develops already below $T^{s}$ as predicted, e.g., in Ref. [34]. Figure 2(c) shows indeed a weak, yet significant increase of $\lambda^{\mathrm{TF}}$ below about $50 \mathrm{~K}$ which is compatible with a precursor SDW that fluctuates on the microsecond time scale (but is quasistatic on the IR time scale). Finally, the decrease of the PBP below $T_{c}=21.7 \mathrm{~K}$ resembles the one of $\lambda^{\mathrm{TF}}$ in Fig. 2(c) and confirms that the SC and SDW orders are competing for the same low-energy states. We remark that this reduction of SW ${ }^{600-1400}$ cannot be directly caused by the formation of the SC gap. This is evident from Fig. 4(b) where $\Delta \sigma_{1}=\sigma_{1}(5 \mathrm{~K})-\sigma_{1}(25 \mathrm{~K})$ for the OP sample (where $\Delta^{\mathrm{SC}}$ is even slightly larger than for the UD one [22]) essentially vanishes above $600 \mathrm{~cm}^{-1}$. As compared to the $\mathrm{SW}$ of the $\mathrm{SC}$ condensate of $\mathrm{SW}^{\delta} \approx 7 * 10^{5} \Omega^{-1} \mathrm{~cm}^{-2}$, the one of 

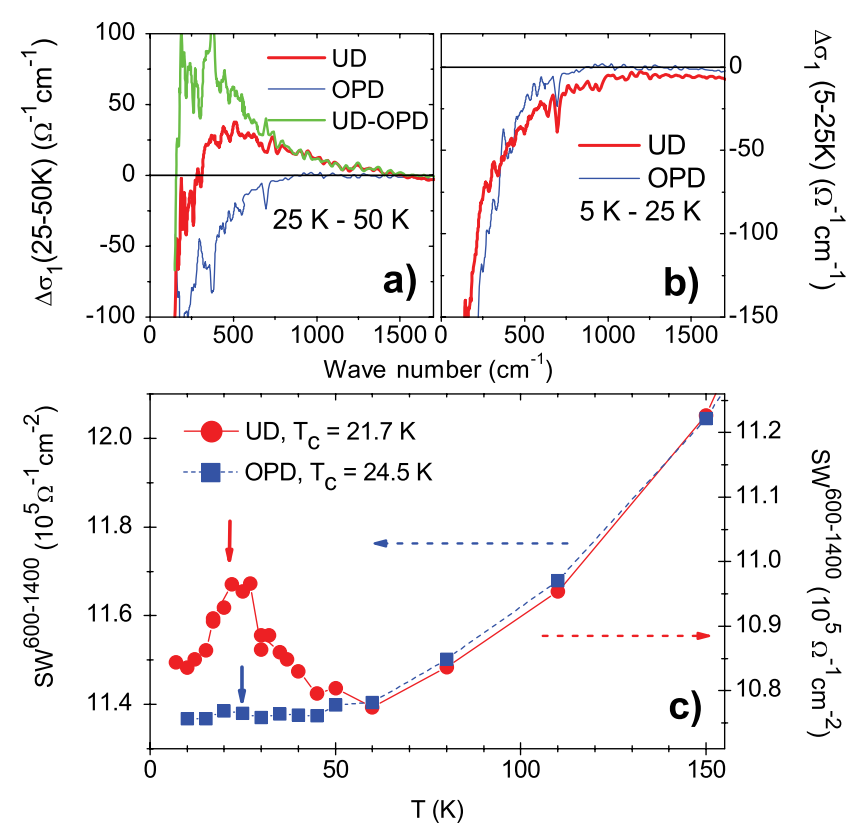

FIG. 4 (color online). $T$-dependent spectral changes for UD $\mathrm{BaFe}_{1.89} \mathrm{Co}_{0.11} \mathrm{As}_{2}$ and OP $\mathrm{BaFe}_{1.87} \mathrm{Co}_{0.13} \mathrm{As}_{2}$ as obtained with IR ellipsometry. (a) and (b) Difference of the optical conductivity between 25 and $50 \mathrm{~K}$ and between 5 and $25 \mathrm{~K}$, respectively. (c) $T$ dependence of the spectral weight between 600 and $1400 \mathrm{~cm}^{-1} . T_{c}$ is marked by the solid arrows. The vertical axes are scaled to show the common trends above $50 \mathrm{~K}$.

the SDW thus amounts to only $\sim 3 \%-4 \%$ above $T_{c}$ and $<2 \%$ at $T \ll T_{c}$. The SDW state, while it occupies the entire sample volume (as shown by $\mu \mathrm{SR}$ ), thus involves only a very small fraction of the electronic states.

These trends are consistent with the scenario of spin fluctuation mediated SC where the competing SDW and SC orders are separated in momentum space [35]. The SDW develops here only in those parts of the Fermi surface where the nesting condition overcomes a critical threshold. These regions are shrinking as a function of Co doping and the SC pairing, which is determined by the averaged spin susceptibility, becomes strongest just as the SDW finally disappears.

In summary, we performed $\mu \mathrm{SR}$ and IR experiments on weakly underdoped $\mathrm{BaFe}_{1.89} \mathrm{Co}_{0.11} \mathrm{As}_{2}$ which establish that superconductivity and static magnetism coexist and compete on the nanometer length scale. In particular, our combined data establish the bulk nature of the superconducting and magnetic orders and show that these compete for the same electronic states. The magnetic order below $T^{\text {mag }} \approx 32 \mathrm{~K}$ is likely due to an incommensurate SDW of which a fluctuating precursor develops already below the structural transition at $T^{s} \approx 50 \mathrm{~K}$. While this SDW occupies the entire sample volume, it involves only a small fraction of the electronic states.

This work was partially performed at the IR beam line of the ANKA synchrotron at FZ Karlsruhe, $D$, and at the Swiss muon source, $\mathrm{S} \mu \mathrm{S}$, at Paul Scherrer Institut,
Villigen, CH. It was financially supported by the Schweizerischer Nationalfonds (SNF) via Grants No. 200020-119784 and No. 200020-129484, the NCCRMaNEP, and the Sciex-NMS ${ }^{\mathrm{CH}}$ Grant No. CZ0908003. A.J.D. acknowledges financial support from the Leverhulme Trust.

*christian.bernhard@unifr.ch

[1] Y. Kamihara, T. Watanabe, M. Hirano, and H. Hosono, J. Am. Chem. Soc. 130, 3296 (2008).

[2] Ch. Niedermayer et al., Phys. Rev. Lett. 80, 3843 (1998).

[3] S. Sanna, G. Allodi, G. Concas, A. D. Hillier, and R. DeRenzi, Phys. Rev. Lett. 93, 207001 (2004).

[4] R. I. Miller et al., Phys. Rev. B 73, 144509 (2006).

[5] Ch. Pfleiderer, Rev. Mod. Phys. 81, 1551 (2009).

[6] C. de la Cruz et al., Nature (London) 453, 899 (2008).

[7] H.H. Klauss et al., Phys. Rev. Lett. 101, 077005 (2008).

[8] N. Ni et al., Phys. Rev. B 78, 214515 (2008).

[9] A. J. Drew et al., Nature Mater. 8, 310 (2009).

[10] D. K. Pratt et al., Phys. Rev. Lett. 103, 087001 (2009).

[11] A. D. Christianson et al., Phys. Rev. Lett. 103, 087002 (2009).

[12] M. H. Julien et al., Europhys. Lett. 87, 37001 (2009).

[13] Y. Laplace, J. Bobroff, F. Rullier-Albenque, D. Colson, and A. Forget, Phys. Rev. B 80, 140501 (2009).

[14] I. I. Mazin, D. J. Singh, M. D. Johannes, and M. H. Du, Phys. Rev. Lett. 101, 057003 (2008).

[15] H. Luetkens et al., Nature Mater. 8, 305 (2009).

[16] Y. J. Uemura, Nature Mater. 8, 253 (2009).

[17] F. Hardy, P. Adelmann, T. Wolf, H. v. Löhneysen, and C. Meingast, Phys. Rev. Lett. 102, 187004 (2009).

[18] C. Lester et al., Phys. Rev. B 79, 144523 (2009).

[19] F. Rullier-Albenque, D. Colson, A. Forget, and H. Alloul, Phys. Rev. Lett. 103, 057001 (2009).

[20] A. Schenck, Muon Spin Rotation: Principles and Applications in Solid State Physics (Adam Hilger, Bristol, 1986).

[21] H. Maeter et al., Phys. Rev. B 80, 094524 (2009).

[22] K. W. Kim et al., Phys. Rev. B 81, 214508 (2010).

[23] D. N. Basov and T. Timusk, Rev. Mod. Phys. 77, 721 (2005).

[24] A. A. Aczel et al., Phys. Rev. B 78, 214503 (2008).

[25] J. E. Sonier et al., Phys. Rev. Lett. 72, 744 (1994).

[26] H. Luetkens et al., Phys. Rev. Lett. 101, 097009 (2008).

[27] A. J. Drew et al., Phys. Rev. Lett. 101, 097010 (2008).

[28] C. Bernhard et al., New J. Phys. 11, 055050 (2009).

[29] E. van Heumen et al., Europhys. Lett. 90, 37005 (2010).

[30] G. Li et al., Phys. Rev. Lett. 101, 107004 (2008).

[31] M. Tinkham, Introduction to Superconductivity (McGrawHill, Singapore, 1996).

[32] M. Dressel and G. Grüner, Electrodynamics of Solids (Cambridge University Press, Cambridge, U.K., 2002).

[33] W.Z. Hu et al., Phys. Rev. Lett. 101, 257005 (2008).

[34] I. I. Mazin and M.D. Johannes, Nature Phys. 5, 141 (2009).

[35] A. B. Vorontsov, M. G. Vavilov, and A. V. Chubukov, Phys. Rev. B 79, 060508(R) (2009). 